\title{
The S6KII ( rsk) Gene of Drosophila melanogaster Differentially Affects an Operant and a Classical Learning Task
}

\author{
Gabriele Putz, ${ }^{1}$ Franco Bertolucci, ${ }^{1}$ Thomas Raabe, ${ }^{2}$ Troy Zars, ${ }^{1}$ and Martin Heisenberg ${ }^{1}$ \\ ${ }^{1}$ Lehrstuhl für Genetik und Neurobiologie, Biozentrum, Am Hubland, D-97074 Wuerzburg, Germany, and ${ }^{2}$ Institut für Medizinische Strahlenkunde und \\ Zellforschung, D-97078 Wuerzburg, Germany
}

\begin{abstract}
In an attempt to dissect classical and operant conditioning in Drosophila melanogaster, we have isolated the gene for ribosomal S6 kinase II (S6KII). This enzyme is part of a family of serine-threonine kinases that in mammals have been implicated in the MAPK (mitogenactivated protein kinase) signaling cascade controlling (among other processes) synaptic plasticity (long-term potentiation/long-term depression) and memory formation. The human homolog $r s k 2$ has been linked to mental retardation (Coffin-Lowry syndrome). Mutant analysis in Drosophila shows that S6KII serves different functions in operant place learning and classical (pavlovian) olfactory conditioning. Whereas in the null mutant only pavlovian olfactory learning is affected, a P-element insertion mutant reducing the amount of S6KII only affects operant place learning. A mutant lacking part of the N-terminal kinase domain and performing poorly in both learning tasks is dominant in the operant paradigm and recessive in the pavlovian paradigm. The behavioral defects in the pavlovian task can be rescued by the genomic S6KII transgene. Overexpression of S6KII in wild type has a dominant-negative effect on the operant task that is rescued by the null mutant, whereas in the pavlovian task overexpression may even enhance learning performance.
\end{abstract}

Key words: Drosophila melanogaster; associative learning; heat box; operant conditioning; classical conditioning; 90 ribosomal S6 kinase

\section{Introduction}

In associative pavlovian (classical) conditioning, the organism exploits the temporal relationships of events in the environment; in operant conditioning, the animal adapts its actions to their consequences. In search of genes in Drosophila melanogaster that would differentially affect the two forms of learning, we have isolated the gene S6KII that encodes ribosomal S6 kinase II (Wassarman et al., 1994).

Ribosomal S6 kinases [RSKs; also known as $p 90^{r s k}$, S6KI and S6KII, or mitogen-activated protein kinase (MAPK)-activated protein kinase-1] are a family of serine-threonine kinases that become activated by and are mediators of the Ras-extracellular signal-regulated kinase (ERK) signaling pathway (Frodin and Gammeltoft, 1999). Four RSK isoforms (RSK1-4) are known in vertebrates, whereas in Drosophila only a single isoform has been

Received July 6, 2004; revised Sept. 8, 2004; accepted Sept. 13, 2004.

This work was supported by the Deutsche Forschungsgemeinschaft (SFB 554; Graduiertenkolleg "Arthropodenverhalten"), the Human Frontiers Science Program (RG 0143), and Fonds der Chemischen Industrie. We thank Dr. U. Schaefer for providing the P-element lines, Dr. E. Hafen for the UAS:S6KIII transgenic lines, Dr. S. Kramer for contribution to the behavioral screen, R. Wolf for assistance throughout this study, K. Oechsner and H. Kaderschabek for maintenance of the heat box, and in particular E. Dierichs-Schmitt for skillful technical help.

The publication costs of this article were defrayed in part by payment of page charges. This article must therefore be hereby marked "advertisement" in accordance with 18 USC section 1734 solely to indicate this fact.

Correspondence should be addressed to Dr. Martin Heisenberg, Lehrstuhl für Genetik und Neurobiologie, Biozentrum, Am Hubland, D97074 Wuerzburg, Germany. E-mail: heisenberg@biozentrum.uni-wuerzburg.de.

G. Putz's present address: Max-Planck Institute of Molecular Cell Biology and Genetics, Pfotenhauerstrasse 108, D-01307 Dresden, Germany.

T. Zars's present address: Division of Biological Sciences, University of Missouri, Columbia, M0 65211

DOI:10.1523/JNEUROSCI.3211-04.2004

Copyright $\odot 2004$ Society for Neuroscience $\quad$ 0270-6474/04/249745-07\$15.00/0 described (Wassarman et al., 1994). (We will keep the name S6KII for the Drosophila gene and refer to the Drosophila enzyme as S6KII.) RSKs are characterized by two kinase domains. The C-terminal kinase domain contributes through autophosphorylation to full activation of the N-terminal kinase domain, which in turn phosphorylates substrates such as the cAMP response element-binding protein (CREB), c-Fos, NF $\kappa \mathrm{B}$ (nuclear factor $\kappa \mathrm{B}) / \mathrm{I} \kappa \mathrm{B} \alpha$ (inhibitor of nuclear factor- $\kappa \mathrm{B}$ ), and estrogen receptor (Bjorbaek et al., 1995; Nebreda and Gavin, 1999).

RSKs have diverse functions. They are important players in cell cycle progression, cell survival, and cytostatic-factor arrest. Additional roles of RSKs include the feedback inhibition of the Ras-ERK pathway and the regulation of protein synthesis by phosphorylation of polyribosomal proteins and glycogen synthase kinase-3 (Frodin and Gammeltoft, 1999; Nebreda and Gavin, 1999; Ballif and Blenis, 2001).

Mutations in the human $r s k 2$ gene are associated with the Coffin-Lowry syndrome (CLS) (Lowry et al., 1971), an X-linked disorder characterized by severe psychomotor retardation, digit and facial dysmorphisms, and progressive skeletal deformations (Young, 1988; Trivier et al., 1996). In CLS fibroblasts, a drastic attenuation in the induced Ser-133 phosphorylation of transcription factor CREB was detected in response to epidermal growth factor stimulation (De Cesare et al., 1998).

The ERK/MAPK cascade is involved in neuronal as well as behavioral plasticity (English and Sweatt, 1997; Impey et al., 1998; Sgambato et al., 1998; Orban et al., 1999), and studies assign the RSKs a role in these processes as the direct link between 
MAPKs and CREB (Xing et al., 1996). Near to nothing is known directly about functions of RSKs in invertebrates (Rintelen et al., 2001). However, the MAPK cascade has been implicated in neuronal plasticity in molluscs (Martin et al., 1997), and in Drosophila, a role of the MAPK cascade in pavlovian memory formation was indicated by a mutant of the leonardo (leo) gene encoding a 14-3-3 family protein (Skoulakis and Davis, 1996).

We report here the first functional study of the rsk homolog in Drosophila, the S6KII gene. Unlike in CLS patients, null mutations in the S6KII gene cause no visible external morphological defects but, in accordance with the human condition and the rsk2 knock-out mouse (Dufresne et al., 2001), impair cognitive functions in that operant place learning and pavlovian olfactory conditioning are affected. Yet, the gene plays distinctly different roles in the two forms of behavioral plasticity.

\section{Materials and Methods}

Flies. A collection of X-chromosomal insertion lines of the $\mathrm{P}[\mathrm{lacW}]$ element was provided by Dr. Ulrich Schäfer (Max-Planck Institute for Biophysical Chemistry, Göttingen, Germany). The insertion site of the $\mathrm{P}[\mathrm{lacW}]$ element in $\operatorname{ign}^{P 1}$ was determined by in situ hybridization on polytene chromosomes. Genomic fragments flanking the P insertion site were recovered by plasmid rescue and sequenced. Precise and imprecise excision lines of P-element line ign ${ }^{P 1}$ were generated by remobilization of the $\mathrm{P}[\mathrm{lacW}]$ with introduction of a stable transposase source (Robertson et al., 1988). The lines were screened by Southern blot for precise excision of the P-element and by PCR for deletions of the S6KII gene locus. Deletions of interesting candidates were characterized by sequencing. Lines were converted to the genetic background of wild-type Berlin by repeatedly outcrossing them to $w^{1118}$ flies on a wild-type Berlin background. For additional analysis, the $w^{+}$gene was recombined onto the $\mathrm{X}$ chromosome. Selection for recombination events was done by PCR. Subsequently, the strains were also crossed to wild-type Canton S (WT-CS) for six generations and made homozygous. According to the genetic background of the tested flies, Drosophila strain $w^{1118}$ or WT-CS was used as a control line. Flies were reared on cornmeal/molasses medium (Guo et al., 1996) in a $16 / 8 \mathrm{hr}$ light/dark cycle at $60 \%$ humidity and $25^{\circ} \mathrm{C}$. Adults were studied at 2-7 d after eclosion.

Operant learning in the heat box. The conditioning apparatus (see Fig. 1) consists of an array of 15 chambers $(26 \times 4 \times 2 \mathrm{~mm})$ operating in parallel, each with Peltier elements on top and bottom allowing for fast heating and cooling (Putz and Heisenberg, 2002). The standard experiment includes a $30 \mathrm{sec}$ pretest, $4 \mathrm{~min}$ training, and $3 \mathrm{~min}$ test period. (In some experiments, the pretest period is extended to $5 \mathrm{~min}$, a measure that may slightly reduce variation in the data.) During the experiment, one half of the chamber is defined as the "punished" side and the other as the "unpunished" side. Designations are altered for every experiment. In the pre-test, the fly can explore the chamber at a constant temperature of $20^{\circ} \mathrm{C}$. During the training period, the whole chamber is heated to $40^{\circ} \mathrm{C}$ whenever the fly enters the punished side and is cooled down to $20^{\circ} \mathrm{C}$ when it enters the unpunished side. In the test period following immediately, the chamber is constantly at $20^{\circ} \mathrm{C}$. A performance index (PI) is calculated as the difference between the time the fly spent in the unpunished versus punished half of the chamber divided by the total time. For analysis, training and test phases were binned into 1 or 2 min blocks. Training values refer to the last minute of training and test values to the first minute of the memory test. Because tests for normal distribution of performance indices yield varying results, nonparametrical tests were used for statistical evaluation. Two independent groups were compared by Mann-Whitney $U$ tests. For comparison of three and more groups, Kruskal-Wallis ANOVA tests were used. Thermo-sensitivity tests were performed as described by Zars (2001).

Olfactory learning. Olfactory memory was tested with a modified version of the conditioning apparatus of Tully and Quinn (1985). In this assay, groups of 50-100 flies are sequentially exposed to two odorants, one of which is paired with electric shocks as a negative reinforcer. During the procedure, wild-type flies learn to prefer the unpunished odorant.

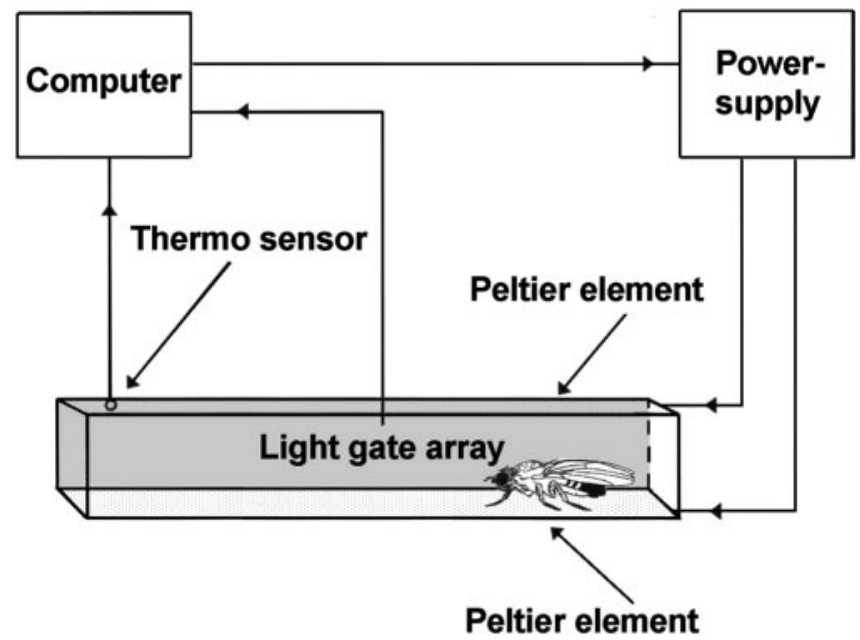

Figure 1. Schematic diagram of one of the 15 modified heat boxes operated in parallel.

The olfactory discrimination learning paradigm, control experiments for shock reactivity and odor perception, as well as calculations, are performed after that described by de-Belle and Heisenberg (1994), with slight modifications. Pure benzaldehyde (100 $\mu$ l, in cups of $5 \mathrm{~mm}$ diameter, $7 \mathrm{~mm}$ depths) and 3-octanol ( $900 \mu \mathrm{l}$, in cups of $16 \mathrm{~mm}$ diameter, 10 $\mathrm{mm}$ depths) are used as odorants. Negative reinforcement consists of 12 $130 \mathrm{~V}$ DC electric shocks over $60 \mathrm{sec}$ (one every $5 \mathrm{sec}$ ) with each shock lasting $1.25 \mathrm{sec}$. Repeated-measures ANOVAs were used for comparison between groups; when warranted, the Duncan post hoc test was performed.

Reverse transcription-PCR. Isolation of poly $(\mathrm{A})^{+}$mRNA from total RNA was obtained by using the Oligotex mRNA Mini kit from Qiagen (Hilden, Germany). SUPERSCRIPT First Strand Synthesis System from Invitrogen (San Diego, CA) was optimized to synthesize first-strand cDNA from varying amounts of purified poly(A) ${ }^{+}$or total RNA. RNA was reverse transcribed using random hexamers. The quality of isolated cDNA was tested by amplification of the rp49 ribosomal gene.

Transgenic lines. The $6.5 \mathrm{~kb}$ genomic fragment used for rescue experiments was derived from BACCR05K22 (AC011760) (Hoskins et al., 2000 ) and was cloned into the pW8 transformation vector (Klemenz et al., 1987). Transgenic lines were generated by injecting Qiagen-purified plasmid DNA into $w^{1118}$ embryos. Six independent transgenic lines were established and cantonized for six generations. The site of plasmid insertion was characterized.

Antibodies and Western blot analysis. A peptide (ADLD-SARKRPTHRTLC) corresponding to amino acids 119-134 of the S6KII protein was synthesized and used to immunize rabbits by a commercial supplier. For Western blot analysis, protein lysates from 10 heads of male or female adult flies of the indicated genotype were separated by SDS-PAGE. As a control, protein extracts from five heads of flies expressing the UAS: S6KII transgene under hs-Gal4 control were used. [Transgenic lines expressing the S6KII gene under Gal4/UAS control were kindly provided by E. Hafen (Zurich, Switzerland) (Rintelen et al., 2001)]. After transfer to nitrocellulose, the blot was incubated overnight at $4^{\circ} \mathrm{C}$ with the affinitypurified anti-S6KII serum (1:500), then probed with an HRP-conjugated secondary antibody and developed using the ECL kit (Amersham Biosciences, Braunschweig, Germany).

\section{Results}

Behavioral and molecular characterization of mutant ign $^{P 1}$

Operant place learning in the heat box (Putz and Heisenberg, 2002) was used to screen 1221 homozygous viable, X-chromosomal P-element insertion $(\mathrm{P}[\mathrm{lacW}])$ lines. Flies were trained to avoid part of an elongated, narrow chamber that was quickly heated from 20 to $40^{\circ} \mathrm{C}$ if the fly entered this part (Fig. 1). In one line called ignorant ${ }^{P 1}$ $\left(i g n^{P 1}\right)$, males showed reduced performance during the training $(\mathrm{tr})$ and in the subsequent memory test (te) (Utest; tr: $Z=2.70, p<0.01$; 


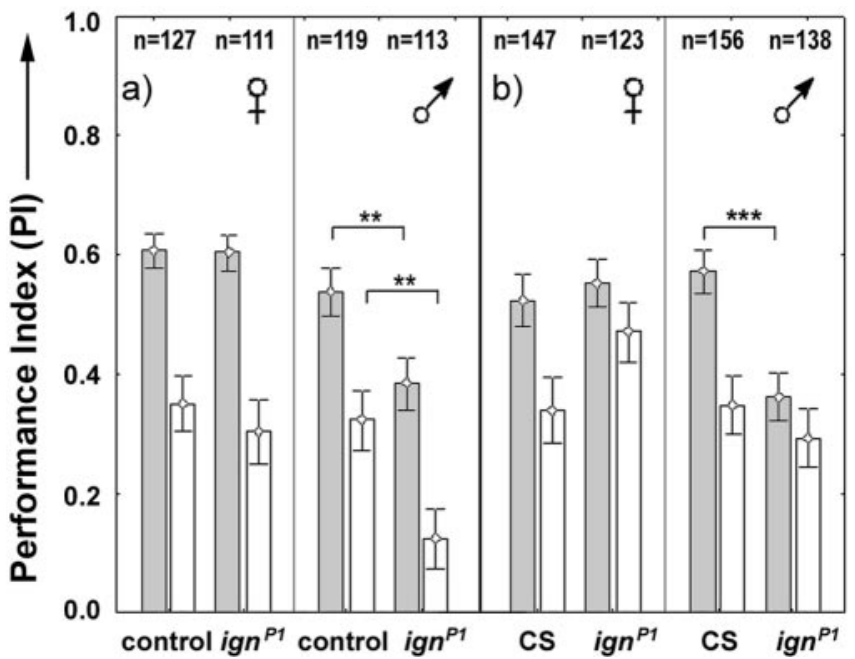

Figure 2. $\quad$ ign $^{p 1}$ mutant shows a male-specific behavioral phenotype in heat-box conditioning. Performance indices of ign $^{P 1}$ versus control flies in the standard experiment are shown. The last minute of training (hatched bars) and 1 min memory test (empty bars) are shown separately for males and females. $a$, Performance of $w^{1118} \mathrm{ign}^{P 1}$ mutant in a wild-type Berlin genetic background compared with $w^{1118}$ (Berlin) control flies. b, Performance of ign ${ }^{p 1}$ mutant after recombining the $56 K / I$ gene with the P-element insertion onto the $X$ chromosome carrying the $w^{+}$gene and backcrossing to WT-CS (designated as WT in all subsequent figures). $n$, Number of flies. The error bars indicate SEMs. ${ }^{* *} p<0.01 ;{ }^{* * *} p<0.001$.

te: $Z=2.70, p<0.01)$. No significant defect was observed in females on any genetic background (against $w^{1118}$ on a wild-type Berlin background; $U$ test; tr: $Z=0.13, p=\mathrm{NS}$; te: $Z=0.35, p=\mathrm{NS}$ ) (Fig. $2 a$ ). After recombining the chromosomal region carrying the P-element onto a WT-CS X chromosome carrying the $w^{+}$gene and extensive additional backcrossing of the autosomes to WT-CS, a robust male-specific defect during the training phase remained ( $U$ test; $Z=3.57, p<0.001$ ), whereas performance in the memory test was close to normal in both genders ( $U$ test; males: $Z=0.87, p=\mathrm{NS}$; females: $Z=-1.43, p=\mathrm{NS}$ ) (Fig. $2 b$ ). Thermo-sensitivity was not impaired in $\operatorname{ign}^{P 1}$ mutant males (repeated-measures ANOVA; males: $F=0.93, p=\mathrm{NS}$; females: $F=0.77, p=\mathrm{NS}$; see supplemental material, available at www.jneurosci.org). Because the memory phenotype of $i g n^{P 1}$ seemed to be under polygenic control and was not apparent in the WT-CS genetic background, we decided to refer, for the rest of this report, only to the performance deficit during training, although in some of the figures first-minute memory scores will be shown as well.

\section{Molecular characterization of $\operatorname{ign}^{P 1}$ and excision lines}

Sequencing of DNA flanking the P-element in the $i g n^{P 1}$ line showed that it was inserted in the $5^{\prime}$ untranslated region of the first exon of the gene coding for S6KII (CG17596) (Fig. 3). The P-element insertion is located $355 \mathrm{bp}$ in front of the predicted translational start site. Sequencing of expressed-sequence tag clones SD05277, GH08264, and GH21818 (Stapleton et al., 2002) confirmed Flybase information about the structure of S6KII. The P-element insertion is located at position 27/28 of clone SD05277. To test whether the P-element insertion in ign ${ }^{P 1}$ flies is responsible for the behavioral defect, and second, to obtain additional S6KII mutants, P-element excision lines of $i g n^{P 1}$ flies were generated. The P-element was remobilized crossing $\operatorname{ign}^{P 1}$ to a source of transposase (Robertson et al., 1988). Based on the loss of the mini- $w^{+}$marker of $\mathrm{P}[\mathrm{lacW}]$, we obtained 128 jump-out lines from F1 females and 227 from F1 males (Engels et al., 1990). In a first screen of jump-outs in females, 13 lines were found by

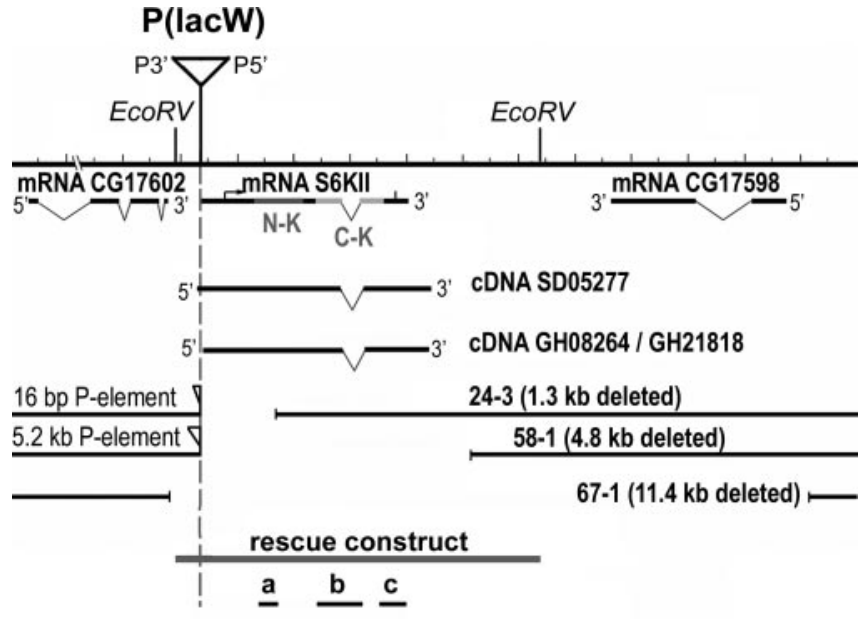

Figure 3. Molecular map of the $S 6 K I /$ gene (partitions, $0.5 \mathrm{~kb}$ ). The following are shown: EcoRV restriction sites; insertion site of $P[\mathrm{lacW}]$ of the $i g n^{P 1}$ mutant; the exon/intron structure of S6KII and predicted neighboring genes; structure of sequenced CDNAs (SD0522, GH21818, and GH08264); extension of deletions; the rescue construct; and the regions amplified by RT-PCR (a, with primers GCGGCAAAATCGTGTCCCTTTC and CTGGATTTTCTTCGTGGCGGTG; $b$, with primers ACCTCGGGAGCCACAAAATTGG and AGCATCCACATCCACTTCTGCC; $c$, with primers ATATAGATGCCCCGCACAG and GCAGCAGAATCACATCTCC). N-K, N-terminal kinase domain; C-K, C-terminal kinase domain.

Southern blot to be candidate precise jump-out lines. Two lines, $i g n^{\Delta l P 1}$ and $i g n^{\Delta 2 P 1}$, restored wild-type sequence at the P-element insertion site. Line $i g n^{\Delta l P 1}$ does not show any nucleotide changes close to this site, whereas line $i g n^{\Delta 2 P 1}$ has several nucleotide changes surrounding the P-element insertion site in the untranslated region. The remaining jump-out lines were screened by PCR, and 13 of them contained deletions larger than $1 \mathrm{~kb}$. Six of them were characterized in more detail. Sequencing of the complete region revealed that they had lost part or all of the S6KII gene (Fig. 3). Deletion line Df(1)ign ${ }^{\Delta 24-3}$ had a loss of $1322 \mathrm{bp}$ of genomic sequence in the $5^{\prime}$ region of the S6KII gene, removing part of the first exon. In a similar mutant, $D f(1) i g n^{\Delta 30-2}, 2197 \mathrm{bp}$ were deleted. In excision lines $D f(1) i g n^{\Delta 67-1}$ and $D f(1) i g n^{\Delta 58-1}$, the coding region of S6KII was completely removed. Lines $D f(1) i g n^{\Delta 24-3}, D f(1) i g n^{\Delta 30-2}$, and $D f(1) i n^{\Delta 58-1}$ still had retained part of the P-element, ranging from $16 \mathrm{bp}$ to $5.3 \mathrm{~kb}$. The largest of the deletions, $D f(1)$ ign $^{\Delta 67-1}$, removed the entire S6KII gene, the $\mathrm{P}$-element, and a neighboring gene encoding a serine-threonine phosphatase (Fig. 3) (CG17598). All deletion lines were homozygous viable. The name ignorant (ign) was retained (as a synonym of S6KII) for all mutants derived from $i g n^{P 1}$.

\section{Expression of S6KII}

To investigate whether $i g n^{P 1}$ and the deficiencies were null mutants, we searched for transcripts by reverse transcription (RT)PCR. As expected, lines $D f(1) i g n^{\Delta 67-1}$, with a deletion of 11,366 bp, and $D f(1) i g n^{\Delta 58-1}(4762 \mathrm{bp})$, both removing all or nearly all of the S6KII gene, were null mutants by this criterion. Deletion lines $D f(1)$ ign $^{\Delta 24-3}$ and $D f(1) i g n^{\Delta 30-2}$ still had S6KII transcript in the regions where genomic DNA was intact. In the original P-element line, the S6KII gene seemed to be fully transcribed because signal was obtained in all investigated genomic regions (see $a-c$ in Fig. 3) (RT-PCR data not shown). To analyze the expression of S6KII protein in wild-type and mutant flies, an antiserum directed against a short peptide from the N-terminal region of the protein was generated and used in Western blots (Fig. 4A). As a control, UAS:S6KII transgenic flies (Rintelen et al., 

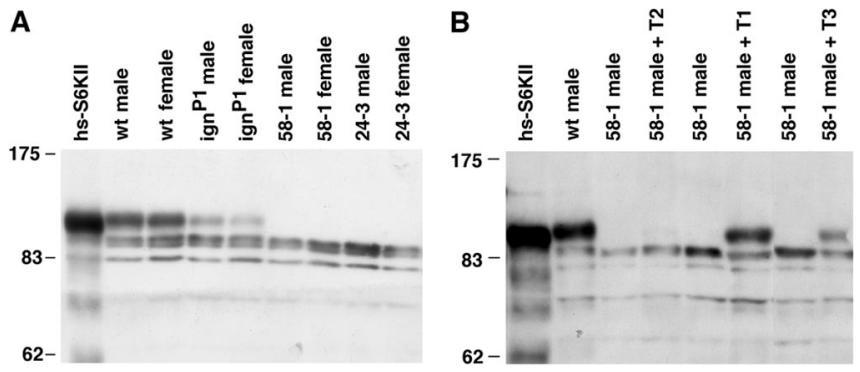

Figure 4. Western blots of WT-CS and mutants. S6KII has a molecular weight of $90 \mathrm{kDa}$ as witnessed by overexpression in hsGAL4/UAS-ign flies (left). $A$, This band has the same intensity in males and females of wild type and is equally reduced in both genders in the P-element line $i g n^{P 1}$. The band is missing in the full deletion $D f(1) \operatorname{ign}^{\Delta 58-1}$, and the partial deletion $D f(1) i^{\Delta n^{\Delta 24-3}}$ is missing the genomic sequence used for antigen production. B, S6KII expression is restored in the male progeny of transgenic lines T1-T3 crossed to mutant Df(1)ign ${ }^{\Delta 8-1}$ females (e.g., 58-1 male + T1). (Note the faint band in the fourth lane.) The bottom bands are unrelated proteins cross-reacting with the anti-S6KII-serum.

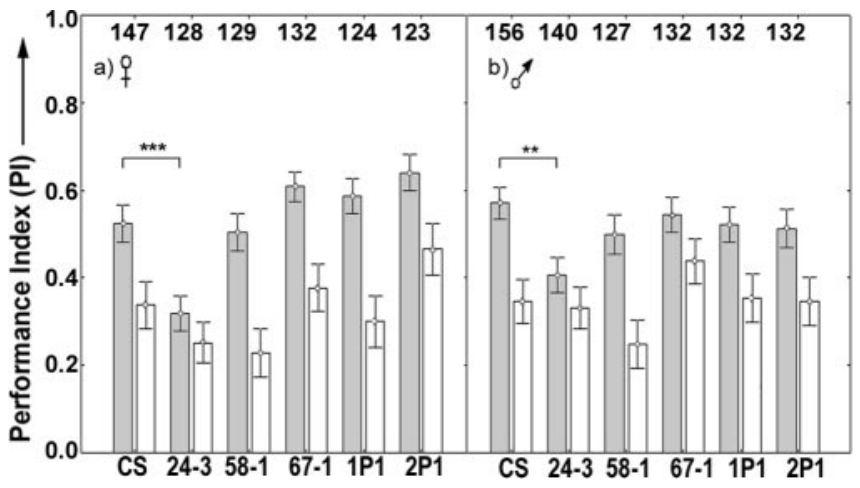

Figure 5. Operant learning of deletion lines versus WT-CS in the heat box standard experiment. Performance indices of the last 1 min training (gray bars) and first 1 min memory (empty bars) periods are shown for females $(a)$ and males $(b)$. The numbers above the bars indicate the number of flies tested. The error bars are SEMs. ${ }^{* *} p<0.01 ;{ }^{* *} p<0.001$.

2001) were crossed to flies carrying a Gal4 transgene under control of the heat shock promoter. After heat shock, high levels of a $90 \mathrm{kDa}$ protein corresponding in size to the predicted molecular weight of S6KII were expressed. A protein of the same molecular weight was detected in wild-type flies but was missing in the deletion line $D f(1) i g n^{\Delta 58-1}$. Unexpectedly, in the mutant $i g n^{P 1}$, S6KII was reduced in both sexes to a similar degree, despite the sexually dimorphic behavior of $i g n^{P 1}$ in operant place learning. As expected, in $D f(1) i g n^{\Delta 24-3}$, no S6KII protein could be detected, because the serum recognized a peptide from the region deleted in the mutant.

\section{Performance of jump-out lines in heat-box conditioning}

Similar to the $\operatorname{ign}^{P 1}$ mutant, some of the excision lines above were backcrossed extensively to WT-CS after recombination of the $w^{+}$ gene onto the $\mathrm{X}$ chromosome. These lines were tested in the heat box (Fig. $5 a, b$ ). Focusing on the last training minute, both males and females of the precise excision line $i g n^{\Delta I P 1}$ performed well ( $U$ test; males: $Z=1.08, p=\mathrm{NS}$; females: $Z=-0.77, p=\mathrm{NS}$ ). The same was found for $i g n^{\Delta 2 P 1}$ ( $U$ test; males: $Z=0.64, p=\mathrm{NS}$; females: $Z=-1.91, p=$ NS). Thus, excision of the P-element reverts the phenotype of $i g n^{P 1}$ flies to wild type.

Flies carrying a deletion of the 5' part of the S6KII gene including the N-terminal kinase domain $\left(D f(1) \operatorname{ign}^{\Delta 24-3} ; D f(1) \operatorname{ign}^{\Delta 30-2}\right.$ ) showed reduced heat-box learning in both genders ( $U$ test; $D f(1)$ ign $^{\Delta 24-3}$, homozygous females: $Z=4.19, p<0.001$; hemi-

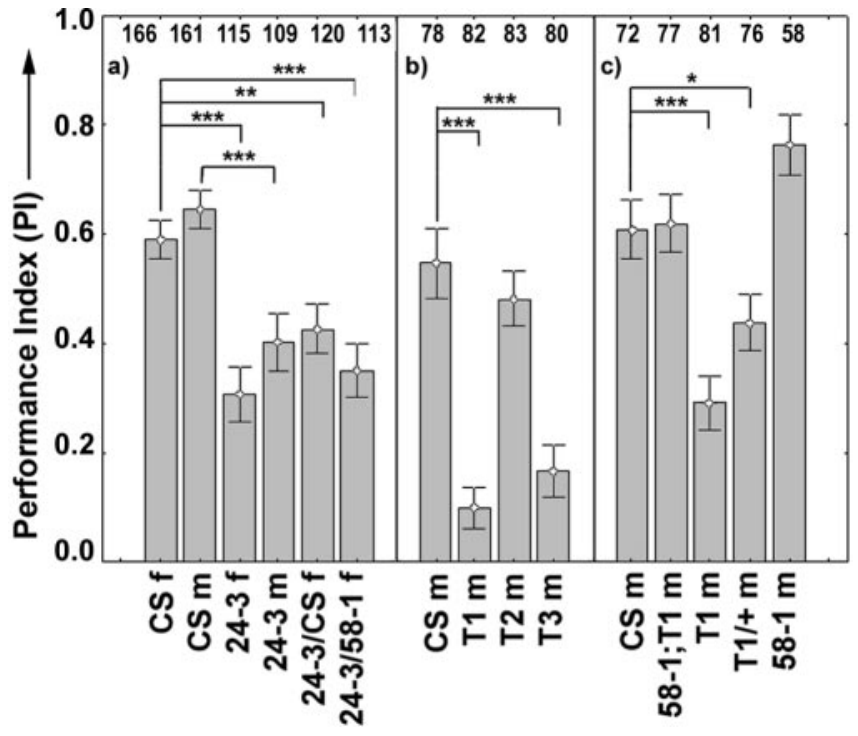

Figure 6. a, Deficit in heat-box learning of partial deletion mutant $D f(1)$ ign $^{\Delta 24-3}$ is dominant. Shown are female $(\mathrm{f})$ and male $(\mathrm{m})$ performance in the last 1 min training period of homozygous and heterozygous mutant flies, as well as double heretozygous Df(1)ign ${ }^{\Delta 24-3}$ / $D f(1)$ ign $^{\Delta 58-1}$ flies versus WT-CS in the standard experiment. $b$, Homozygous transgenic lines expressing two copies of S6KII show reduced operant learning in the heat box. (Compare expression levels of T1-T3 in Fig. 4.) c, Rescue of operant learning if expression of transgene (T1) is compensated by the deletion of the endogenous $56 \mathrm{~K} / \mathrm{g}$ gene. Note that the PI of T1/T1 is higher than in $b$ because of a different protocol. The numbers above the bars indicate the number of flies tested. The error bars are SEMs. ${ }^{*} p<0.05 ;{ }^{* *} p<0.01 ;{ }^{* * *} p<0.001$.

zygous males: $Z=3.51, p<0.001$; data for $D f(1)$ ign $^{\Delta 30-2}$ are shown in supplemental material, available at www.jneurosci. org). Crossing $D f(1)$ ign $^{\Delta 24-3}$ to the larger-deficiency $D f(1) R 29$ (see Flybase) deleting the whole region of the S6KII gene gave similar results: $D f(1) R 29 / D f(1) i g n^{\Delta 24-3}$ flies had a reduced learning score (F. Schulz, B. Poeck, and M. Heisenberg, unpublished results). Also, trans-heterozygous $D f(1) i_{i g n^{\Delta 24-3} / D f(1) i g n^{\Delta 58-1}}$ flies showed reduced learning ( $U$ test; $Z=3.69, p<0.001$ ) (Fig. $6 a$ ) indistinguishable from that of the homozygous $D f(1) i_{g} n^{\Delta 24-3}$ (Fig. 6a). Surprisingly, the defect was fully dominant because heterozygous females $\left(D f(1) \operatorname{ign}^{\Delta 24-3} /+\right)$ performed as poorly as homozygous mutant females and hemizygous mutant males (heterozygous females: $U$ test: $Z=2.73, p<0.01$ ) (Fig. $6 a$ ).

The next surprise came with $D f(1) i g n^{\Delta 58-1}$. Removal of the complete coding region did not affect operant conditioning in the heat box ( $U$ test; males: $Z=1.24, p=\mathrm{NS}$; females: $Z=0.34$, $p=\mathrm{NS}$ ). Even the removal of $11.3 \mathrm{~kb}$ extending to both sides of the P-element insertion site and including S6KII and a neighboring putative phosphatase gene (CG17598) in $D f(1)$ ign $^{\Delta 67-1}$ did not lead to a defect in performance ( $U$ test; males: $Z=0.90, p=$ NS; females: $Z=-0.73, p=$ NS), implying that the wild-type function of the S6KII gene is not essential for heat-box learning. This further implies that the small deletions $D f(1) i g n^{\Delta 24-3}$ and $D f(1) i g n^{\Delta 30-2}$, as well as $i g n^{P 1}$, are gain-of-function mutants.

To exclude the possibility that thermo-reception was impaired in any of the lines with reduced performance, flies were tested in a thermo-sensitivity assay. Neither in males (repeatedmeasures ANOVA; $F=0.93, p=$ NS) nor in females (repeatedmeasures ANOVA; $F=0.77, p=N S$ ) was a defect in thermoreception found for any of the investigated lines, supporting the idea that the observed low performance in operant conditioning reveals a learning deficit (see supplemental material, available at www.jneurosci.org). 
Transgenic expression of S6KII interferes with place learning We cloned a $6.5 \mathrm{~kb}$ genomic fragment encompassing only the S6KII transcription unit, into a transformation vector (Fig. 3), and established six independent transgenic lines with single insertions (T1-T6). All were homozygous viable. Males from three transgenic lines were crossed to $D f(1) i g n^{\Delta 58-1}$ females, and the male progeny was analyzed on Western blots for expression of S6KII. In all cases, the transgene restored expression of S6KII. In two lines (T1, T3), the amount was similar to that in wild type, and in one (T2), it was distinctly less (Fig. 4B).

All six lines were repeatedly back-crossed to WT-CS and tested in heat-box conditioning. In all lines, females were impaired. In four of them, performance indices during training and test were close to zero (see supplemental material, available at www.jneurosci.org). In males, impairment was slightly less, with mutant phenotypes in only four of the six stocks (three shown in Fig. $6 b$ ) ( $U$ test; T1 males: $Z=6.14, p<0.001$; T2 males: $Z=1.64$, $p=\mathrm{NS}$; T3 males: $Z=5.15, p<0.001$; full data in supplemental material, available at www.jneurosci.org). Expression levels in the three lines tested in Western blots approximately paralleled the behavioral defects (compare Figs. $4 B, 6 b$ ). Moreover, the effect was dose dependent: A single copy of the transgene (T1) was still effective but suppressed the performance less than two copies $(U$ test; T1/+ males: $Z=2.30, p<0.05$ ) (Fig. $6 c$ ).

$\mathrm{T} 1$ was crossed to various S6KII mutants. $D f(1) i g n^{\Delta 58-1} / \mathrm{Y}$; $\mathrm{T} 1 /+$ males carrying a single dose of S6KII as a transgene showed normal training performance in the range of WT-CS and $D f(1)$ ign $^{\Delta 58-1}$ ( $U$ test, $Z=-0.63, p=$ NS) (Fig. $6 c$ ). This indicates that loss of the endogenous S6KII gene can compensate for the dominant-negative effect of the transgene, suggesting that the latter is attributable to dosage rather than tissue specificity (i.e., a modified expression pattern of the transgene).

\section{Pavlovian olfactory conditioning}

Not only place learning in the heat box, but also olfactory discrimination learning (Tully and Quinn, 1985), was affected by mutations in the S6KII gene. Df(1)ign ${ }^{\Delta 58-1}$ females and males showed a reduction of $\sim 40 \%$ in $3 \mathrm{~min}, 30 \mathrm{~min}$, and $3 \mathrm{hr}$ memory compared with WT-CS flies (not separated for gender in Fig. 7, because no sexual differences could be found in any of the data; controls are presented as supplemental material, available at www.jneurosci.org). Independent of sex, the performance of mutant $i g n^{P 1}$ flies in olfactory conditioning was not distinguishable from wild type during the first $3 \mathrm{hr}$, although memory scores tended to be lower in all experiments. The small-deficiency $D f(1)$ ign $^{\Delta 24-3}$ caused $40 \%$ reduction in 3 min memory in both genders (Fig. 8 for males) (data not shown for females). Heterozygous $D f(1) i g n^{\Delta 24-3} /+$ flies performed distinctly better than homozygous mutant flies and were not significantly different from wild type $\left(\mathrm{PI}_{24-3 /+}=61.3+/-6.1 ; n=6 ; p<0.001\right.$ for comparison with homozygous $D f(1)$ ign $^{\Delta 24-3} ; p=$ NS for comparison with WT-CS). Hence, the three kinds of mutants had different effects in the two learning paradigms. The full deletion caused a defect only in olfactory but not in place learning. Inversely, ign ${ }^{P 1}$ males were approximately normal in olfactory but impaired in place learning, and, finally, the partial deletion was a recessive mutation in olfactory and a dominant gain-of-function mutation in place learning.

\section{Genomic rescue in olfactory conditioning}

Homozygous flies of the transgenic line T1 with two extra copies of the gene originally showed significantly better 3 min memory in olfactory conditioning than WT-CS flies (T1/T1: PI $=0.71$,

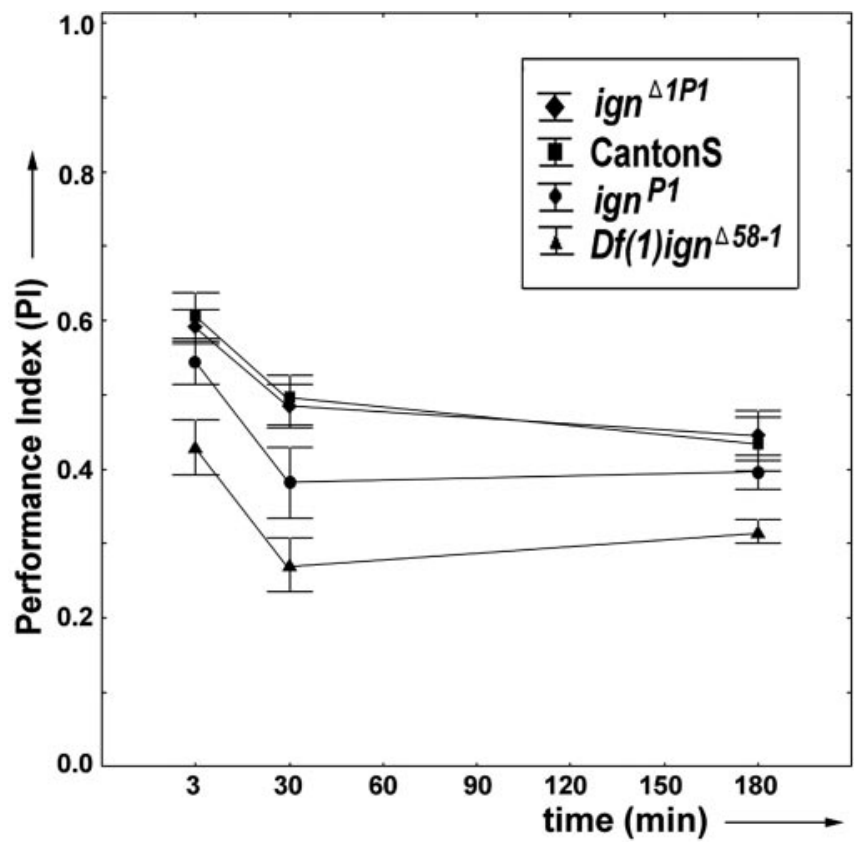

Figure 7. Pavlovian olfactory discrimination learning is impaired in the S6KIInull mutant but not significantly in $i^{p 1}{ }^{p 1}$. The $3 \mathrm{~min}, 30 \mathrm{~min}$, and $3 \mathrm{hr}$ memory scores are shown. Data for males and females are pooled because no statistically significant difference for gender was found. In the line $i g n^{\Delta \backslash P 1}$, the P-element is precisely eliminated by jump-out.

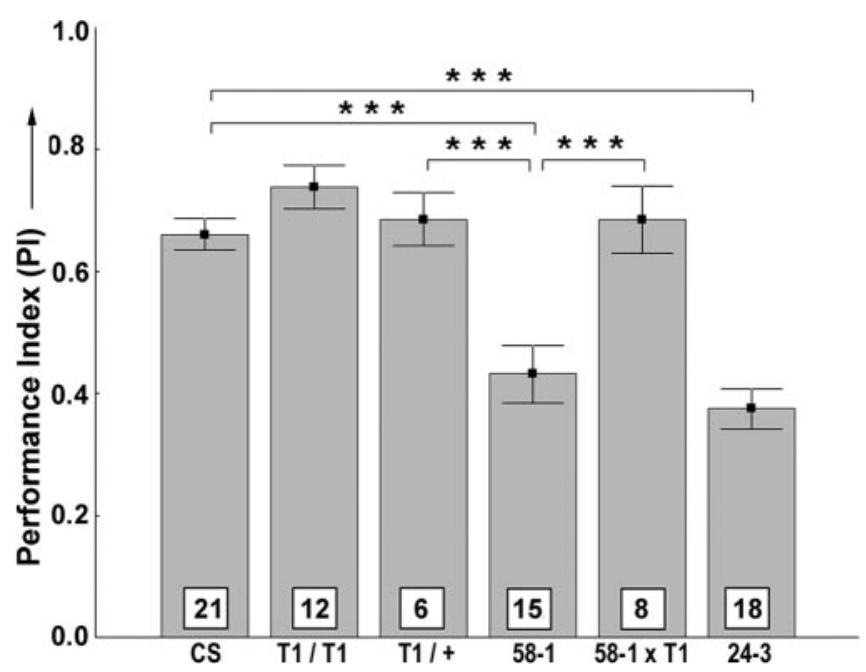

Figure 8. Memory defects of the null mutant $\left[D f(1) \operatorname{ign}^{\Delta 58-1}\right]$ and the partial deletion $\left[D f(1)\right.$ ign $\left.^{\Delta 24-3}\right]$ in pavlovian olfactory conditioning are shown. The defect in $D f(1)$ ign $^{\Delta 58-1}$ is rescued by one copy of the transgene (T1). For technical reasons, only data of males are shown. The error bars indicate SEMs. ${ }^{* *} p<0.001$.

$n=6$; WT-CS: PI $=0.62, n=14 ; p<0.05)$. When this experiment was repeated $\sim 10$ generations later, the difference was marginal and not significant (T1/T1: PI $=0.77, n=6$; WT-CS: PI $=$ $0.75, n=7 ; p>0.05$ ) (data pooled in Fig. 8), leaving the possibility that selection on the genetic background (de-Belle and Heisenberg, 1994) meanwhile had abolished the effect. Whether or not this was the case, the result again contrasted the deleterious effect of the transgene on place learning (Fig. $6 b, c$ ). Moreover, the olfactory learning defect of the deficiency could be rescued by the transgene. $D f(1)$ ign $^{\Delta 58-1} / \mathrm{Y} ; \mathrm{T} 1 /+$ males learned significantly better than $D f(1)$ ign $^{\Delta 58-1}$ flies without the transgene and were indistinguishable from the wild-type controls (Fig. 8). The same was 
found for the partial deletion $D f(1) i g n^{\Delta 24-3}$ (supplemental material, available at www.jneurosci.org).

\section{Discussion}

The Drosophila S6KII is, perhaps surprisingly, encoded by a nonessential gene. Null mutants are viable and normal by morphological criteria and casual behavioral observation. Also, brain structure seems not to be severely affected. As the only apparent defect, $D f(1) i g n^{\Delta 58-1}$ shows a $40 \%$ reduced memory score in pavlovian olfactory learning. Regarding this memory component, the properties of the S6KII mutants are in line with what one might expect from S6KII in vertebrates (Bjorbaek et al., 1995; Nebreda and Gavin, 1999). If it is the kinase domain in the $\mathrm{N}$-terminal part of the enzyme that is phosphorylating target proteins, deletion of that domain should abolish phosphorylation. Indeed, in the mutant $D f(1)$ ign $^{\Delta 24-3}$, lacking part of the $\mathrm{N}$-terminal kinase domain olfactory memory is reduced as much as in the null mutant $\left[D f(1) \operatorname{ign}^{\Delta 58-1}\right]$. The small and the large deletions are recessive (data for $D f(1) i g n^{\Delta 58-1} /+$ are shown as supplemental material, available at www.jneurosci.org), indicating that 50\% S6KII enzymatic activity in the heterozygote is sufficient to provide the full function. In the mutant $i g n^{P I}$, again the reduced overall amount of protein is enough to allow for near to normal learning/memory. Also, the transgene in the genetic background of the null mutant or the partial deletion $D f(1)$ ign $^{\Delta 24-3}$ (data not shown) provides enough enzyme to fully support the behavioral task. Finally, overexpression of S6KII as a transgene has no deleterious effect. If at all, it enhances memory.

The involvement of the S6KII gene in operant place learning could hardly be more different. In wild-type Drosophila, S6KII is not essential for place learning, because removing the whole gene has no apparent phenotype. An influence of the S6KII gene on heat-box learning is seen only in mutants that leave the gene or part of it intact. The $\mathrm{P}$ insertion, the $\mathrm{N}$-terminally truncated gene, and the S6KII transgenes all reduce learning performance. In the $\mathrm{P}$-insertion mutant, the learning defect seems not to be related to the overall amount of S6KII because females learn well and show the same reduced amount of S6KII-like immunoreactivity as males. The heat-box phenotypes need to be studied in detail to find out whether the three kinds of mutations ( $\mathrm{P}$ insertion, partial deletions, overexpression) interfere with heat-box learning in the same manner. Whether S6KII exerts its various effects in adulthood or during development can now be addressed using conditional expression systems (McGuire et al., 2003; Mao et al., 2004).

The S6KII gene is nested in a large intron of a putative gene (CG17602) of unknown function. It should be noted that the mutant phenotypes cannot be assigned to this nearby gene because the genomic transgenes do not contain CG17602 and are inserted far away from the original genomic location. Their phenotypes correspond to and even interact with those of the deficiency mutants.

Assuming that it is at the protein level in which S6KII exerts its deleterious effect on heat-box learning in the mutants, a smaller protein must be synthesized from the C-terminal part of the S6KII gene in the small-deletion mutants. If this is true for the small deletions, it may well be true also for the wild type. This hypothetical small isoform might be mostly suppressed by the large isoform in wild type. Kinases with a large and a small isoform are not uncommon, e.g., in the protein kinase $\mathrm{C}$ family (Sacktor et al., 1993; Drier et al., 2002; Hernandez et al., 2003). It is too early to speculate about mechanisms before a small isoform is found.

Both olfactory learning and place learning require cAMP sig- naling (Tully and Quinn, 1985; Davis et al., 1995; Wustmann et al., 1996) but have their cAMP-dependent memory traces in different neurons (Zars et al., 2000a,b). Because it is now well established that the memory trace for olfactory learning is localized in sets of intrinsic neurons (Kenyon cells) of the mushroom bodies, it will be of interest to determine, by the same reconstitution approach, whether the molecular functions of S6KII documented here colocalize in the same cells. For operant place learning, the localization of the memory trace is not as clear cut as in olfactory learning because several groups of neurons are still candidate locations for the memory trace. However, using the reconstitution strategy, it can be determined whether the same GAL4 lines that rescue in the case of rutabaga also do so with S6KII-cDNA in $D f(1) \operatorname{ign}^{\Delta 58-1}$.

Current schemes for vertebrates place cAMP upstream of S6KII in the same signaling pathway (Impey et al., 1999). This may hold true in Drosophila for pavlovian olfactory conditioning, whereas for operant place learning, S6KII may not lie in the direct pathway (because it is dispensable) but rather in a side branch. With the phenotypes of the mutants and overexpression lines, one is inclined to assume that a close interaction partner or a protein similar to S6KII should be directly involved in place learning. For instance, the signaling pathways for the two learning tasks might diverge at the level of the MAPK that could be blocked by an excess of S6KII and, in particular, by the hypothetical small C-terminal isoform of S6KII. Recent studies in Xenopus oocytes already showed that an N-terminally truncated RSK mutant can constitutively interact with ERK (Gavin et al., 1999; Biondi and Nebreda, 2003).

We do not know whether S6KII in Drosophila is, as in vertebrates, part of the Raf-Ras pathway. As mentioned in Introduction, mutant studies on the Drosophila gene leonardo encoding a 14-3-3 protein (Skoulakis and Davis, 1996) suggest a role for Raf/Ras signaling in olfactory learning and memory. Yet, if S6KII would, as shown in vertebrates, exert its function by phosphorylating CREB or some other transcription factors (Impey et al., 1999), it could not be involved directly in synaptic plasticity. The defect in olfactory learning in the S6KII mutants is apparent already within $3 \mathrm{~min}$, much too early to reflect transcriptional control induced by the learning process. This does not prove an involvement in the modulation of synaptic efficacy, though. A role of the Raf-Ras pathway in the formation and decay of synapses has been demonstrated for the Drosophila neuromuscular junction (Koh et al., 2002). This may be the way in which the leonardo gene affects olfactory learning and memory. This gene has been shown to be required during adulthood (Philip et al., 2001). Yet, the rescue experiments using a heat shock GAL4 driver left a time window of several hours between heat induction and memory test, which would be enough to restore, for instance, the abundance of synapses in a mutant with reduced synaptic density.

Our assessment of S6KII function in Drosophila has focused on learning and memory. This does not exclude that the gene might be involved in other important processes as well. Also, future work will have to show whether the differential effects of the mutants on operant and classical conditioning can be generalized to other operant and classical learning tasks. The study, therefore, cannot claim to have shown that operant and classical associative processes require different biochemical mechanisms. Yet, the strikingly different mutant phenotypes of Drosophila S6KII in operant place learning and pavlovian olfactory conditioning may help to address this question. 


\section{References}

Ballif BA, Blenis J (2001) Molecular mechanisms mediating mammalian mitogen-activated protein kinase (MAPK) kinase (MEK)-MAPK cell survival signals. Cell Growth Differ 12:397-408.

Biondi RM, Nebreda AR (2003) Signalling specificity of Ser/Thr protein kinases through docking-site-mediated interactions. Biochem J 372:1-13.

Bjorbaek C, Zhao Y, Moller DE (1995) Divergent functional roles for p90rsk kinase domains. J Biol Chem 270:18848-18852.

Davis RL, Cherry J, Dauwalder B, Han PL, Skoulakis E (1995) The cyclic AMP system and Drosophila learning. Mol Cell Biochem 149-150:271-278.

de-Belle JS, Heisenberg M (1994) Associative odor learning in Drosophila abolished by chemical ablation of mushroom bodies. Science 263:692-695.

De Cesare D, Jacquot S, Hanauer A, Sassone-Corsi P (1998) Rsk-2 activity is necessary for epidermal growth factor-induced phosphorylation of CREB protein and transcription of c-fos gene. Proc Natl Acad Sci USA 95:12202-12207.

Drier EA, Tello MK, Cowan M, Wu P, Blace N, Sacktor TC, Yin JC (2002) Memory enhancement and formation by atypical PKM activity in Drosophila melanogaster. Nat Neurosci 5:316-324.

Dufresne SD, Bjorbaek C, El-Haschimi K, Zhao Y, Aschenbach WG, Moller DE, Goodyear LJ (2001) Altered extracellular signal-regulated kinase signaling and glycogen metabolism in skeletal muscle from $\mathrm{p} 90$ ribosomal S6 kinase 2 knockout mice. Mol Cell Biol 21:81-87.

Engels WR, Johnson-Schlitz DM, Eggleston WB, Sved J (1990) Highfrequency $\mathrm{P}$ element loss in Drosophila is homolog dependent. Cell 62:515-525.

English JD, Sweatt JD (1997) A requirement for the mitogen-activated protein kinase cascade in hippocampal long term potentiation. J Biol Chem 272:19103-19106.

Frodin M, Gammeltoft S (1999) Role and regulation of 90 kDa ribosomal S6 kinase (RSK) in signal transduction. Mol Cell Endocrinol 151:65-77.

Gavin AC, Ni Ainle A, Chierici E, Jones M, Nebreda AR (1999) A p90(rsk) mutant constitutively interacting with MAP kinase uncouples MAP kinase from p34(cdc2)/cyclin B activation in Xenopus oocytes. Mol Biol Cell 10:2971-2986.

Guo A, Li L, Xia SZ, Feng CH, Wolf R, Heisenberg M (1996) Conditioned visual flight orientation in Drosophila: dependence on age, practice, and diet. Learn Mem 3:49-59.

Hernandez AI, Blace N, Crary JF, Serrano PA, Leitges M, Libien JM, Weinstein G, Tcherapanov A, Sacktor TC (2003) Protein kinase M zeta synthesis from a brain mRNA encoding an independent protein kinase C zeta catalytic domain. Implications for the molecular mechanism of memory. J Biol Chem 278:40305-40316.

Hoskins RA, Nelson CR, Berman BP, Laverty TR, George RA, Ciesiolka L, Naeemuddin M, Arenson AD, Durbin J, David RG, Tabor PE, Bailey MR, DeShazo DR, Catanese J, Mammoser A, Osoegawa K, de Jong PJ, Celniker SE, Gibbs RA, Rubin GM, Scherer SE (2000) A BAC-based physical map of the major autosomes of Drosophila melanogaster. Science 287:2271-2274.

Impey S, Obrietan K, Wong ST, Poser S, Yano S, Wayman G, Deloulme JC, Chan G, Storm DR (1998) Cross talk between ERK and PKA is required for $\mathrm{Ca}^{2+}$ stimulation of CREB-dependent transcription and ERK nuclear translocation. Neuron 21:869-883.

Impey S, Obrietan K, Storm DR (1999) Making new connections: role of ERK/MAP kinase signaling in neuronal plasticity. Neuron 23:11-14.

Klemenz R, Weber U, Gehring WJ (1987) The white gene as a marker in a new P-element vector for gene transfer in Drosophila. Nucleic Acids Res 15:3947-3959.

Koh YH, Ruiz-Canada C, Gorczyca M, Budnik V (2002) The Ras1-mitogenactivated protein kinase signal transduction pathway regulates synaptic plasticity through fasciclin II-mediated cell adhesion. J Neurosci 22:2496-2504.

Lowry B, Miller JR, Fraser FC (1971) A new dominant gene mental retardation syndrome. Association with small stature, tapering fingers, characteristic facies, and possible hydrocephalus. Am J Dis Child 121:496-500.

Mao Z, Roman G, Zong L, Davis RL (2004) Pharmacogenetic rescue in time and space of the rutabaga memory impairment by using Gene-Switch. Proc Natl Acad Sci USA 101:198-203.

Martin KC, Michael D, Rose JC, Barad M, Casadio A, Zhu H, Kandel ER (1997) MAP kinase translocates into the nucleus of the presynaptic cell and is required for long-term facilitation in Aplysia. Neuron 18:899-912.

McGuire SE, Le PT, Osborn AJ, Matsumoto K, Davis RL (2003) Spatiotemporal rescue of memory dysfunction in Drosophila. Science 302:1765-1768

Nebreda AR, Gavin AC (1999) Perspectives: signal transduction. Cell survival demands some Rsk. Science 286:1309-1310.

Orban PC, Chapman PF, Brambilla R (1999) Is the Ras-MAPK signalling pathway necessary for long-term memory formation? Trends Neurosci 22:38-44.

Philip N, Acevedo SF, Skoulakis EM (2001) Conditional rescue of olfactory learning and memory defects in mutants of the 14-3-3zeta gene leonardo. J Neurosci 21:8417-8425.

Putz G, Heisenberg M (2002) Memories in Drosophila heat-box learning. Learn Mem 9:349-359.

Rintelen F, Stocker H, Thomas G, Hafen E (2001) PDK1 regulates growth through Akt and S6K in Drosophila. Proc Natl Acad Sci USA 98:15020-15025.

Robertson HM, Preston CR, Phillis RW, Johnson-Schlitz DM, Benz WK, Engels WR (1988) A stable genomic source of P element transposase in Drosophila melanogaster. Genetics 118:461-470.

Sacktor TC, Osten P, Valsamis H, Jiang X, Naik MU, Sublette E (1993) Persistent activation of the zeta isoform of protein kinase $\mathrm{C}$ in the maintenance of long-term potentiation. Proc Natl Acad Sci USA 90:8342-8346.

Sgambato V, Pages C, Rogard M, Besson MJ, Caboche J (1998) Extracellular signal-regulated kinase (ERK) controls immediate early gene induction on corticostriatal stimulation. J Neurosci 18:8814-8825.

Skoulakis EM, Davis RL (1996) Olfactory learning deficits in mutants for leonardo, a Drosophila gene encoding a 14-3-3 protein. Neuron 17:931-944.

Stapleton M, Carlson J, Brokstein P, Yu C, Champe M, George R, Guarin H, Kronmiller B, Pacleb J, Park S, Wan K, Rubin GM, Celniker SE (2002) A Drosophila full-length cDNA resource. Genome Biol 3:RESEARCH0080.

Trivier E, De Cesare D, Jacquot S, Pannetier S, Zackai E, Young I, Mandel JL, Sassone-Corsi P, Hanauer A (1996) Mutations in the kinase Rsk-2 associated with Coffin-Lowry syndrome. Nature 384:567-570.

Tully T, Quinn WG (1985) Classical conditioning and retention in normal and mutant Drosophila melanogaster. J Comp Physiol [A] 157:263-277.

Wassarman DA, Solomon NM, Rubin GM (1994) The Drosophila melanogaster ribosomal S6 kinase II-encoding sequence. Gene 144:309-310.

Wustmann G, Rein K, Wolf R, Heisenberg M (1996) A jew paradigm for operant conditioning of Drosophila melanogaster. J Comp Physiol [A] 179:429-436

Xing J, Ginty DD, Greenberg ME (1996) Coupling of the RAS-MAPK pathway to gene activation by RSK2, a growth factor-regulated CREB kinase. Science 273:959-963.

Young ID (1988) The Coffin-Lowry syndrome. J Med Genet 25:344-348.

Zars T (2001) Two thermosensors in Drosophila have different behavioral functions. J Comp Physiol [A] 187:235-242.

Zars T, Fischer M, Schulz R, Heisenberg M (2000a) Localization of a shortterm memory in Drosophila. Science 288:672-675.

Zars T, Wolf R, Davis R, Heisenberg M (2000b) Tissue-specific expression of a type I adenylyl cyclase rescues the rutabaga mutant memory defect: in search of the engram [In Process Citation]. Learn Mem 7:18-31. 